\title{
Forecasting the Polish Zloty with Non-Linear Models
}

\author{
Michał Rubaszek, Paweł Skrzypczyński†, Grzegorz Koloch
}

Submitted: 17.08.2010, Accepted: 3.11.2010

\begin{abstract}
The literature on exchange rate forecasting is vast. Many researchers have tested whether implications of theoretical economic models or the use of advanced econometric techniques can help explain future movements in exchange rates. The results of the empirical studies for major world currencies show that forecasts from a naive random walk tend to be comparable or even better than forecasts from more sophisticated models. In the case of the Polish zloty, the discussion in the literature on exchange rate forecasting is scarce. This article fills this gap by testing whether non-linear time series models are able to generate forecasts for the nominal exchange rate of the Polish zloty that are more accurate than forecasts from a random walk. Our results confirm the main findings from the literature, namely that it is difficult to outperform a naive random walk in exchange rate forecasting contest.
\end{abstract}

Keywords: exchange rate forecasting, Polish zloty, Markov-switching models, artificial neural networks.

JEL Classification: C22, C45, C53, F31, G17.

\footnotetext{
*National Bank of Poland, Warsaw School of Economics, e-mail: michal.rubaszek@nbp.pl ${ }^{\dagger}$ National Bank of Poland, e-mail: pawel.skrzypczynski@nbp.pl

${ }^{\ddagger}$ National Bank of Poland, Warsaw School of Economics, e-mail: grzegorz.koloch@nbp.pl
} 


\section{Introduction}

Starting from the seminal article by Meese and Rogoff (1983), which shows that monetary models cannot outperform a naive random walk in out-of-sample exchange rate forecasting, many authors investigated whether it is possible to forecast the future movements of exchange rates at all. The empirical work evolved in many directions: other economic models were tested, different econometric techniques were used and analyses were conducted for various currencies, time samples or data frequencies. The relevant literature can be divided arbitrarily into two lines of research, where the classification depends on whether the emphasis was put on the underlying economic theory or econometric techniques applied in the analysis.

The first strand of the literature tested whether the use of information about macroeconomic fundamentals can improve the accuracy of exchange rate forecasts. The early attempts to reverse the findings of Meese and Rogoff (MR) turned into the use of time-varying coefficients models, the solution which actually was proposed by MR in the conclusions of their article. It was found that this adjustment for parameters instability was not improving the forecasting performance of monetary models, which only strengthened the general belief that exchange rates are not predictable; see e.g. Wolff (1987), Canova (1993). The wisdom that macroeconomic models of nominal exchange rate produce forecasts of poor quality was prevailing till the mid 1990s, when Mark (1995) and Chinn and Meese (1995) showed that model-based forecasts are less accurate than those from a random walk only for short-term horizons. In the case of longer-term forecast horizons, i.e. more than one year, information about the deviation of the exchange rate from its fundamental value can be used to produce exchange rate forecasts that are significantly better than no change forecasts. The belief in the possibility to forecast exchange rates for longer-term horizons was, however, short-lived. The reliability of Mark (1995) results was soon questioned with respect to three major areas. First, Berkowitz and Giorgianni (2001) pointed out that the assumption about cointegration of the exchange rate with fundamentals was not thoroughly tested. Second, Kilian (1999) questioned the robustness of the results with respect to a change in a time or country sample. Third, Faust, Rogers, Wright (2003) were arguing that the analysis was based on the latest-available dataset, whereas macroeconomic data are subject to often and sometimes sizeable revisions. From the numerous other works that attempt to forecast exchange rates using information about macroeconomic fundamentals it is worthy to refer to a comprehensive study by Cheung, Chinn, Pascual (2005). The authors examined the performance of the most popular exchange rate models (the monetary sticky-price model, the interest rate parity model, the Balassa-Samuelson model and the behavioural equilibrium exchange rate model) for many exchange rates and time periods. The main finding of the study is that no model is able to consistently outperform a random walk in exchange rate forecasting. The general conclusion of the above literature is that forecasts conditional on observed macroeconomic fundamentals are dominated by no change forecasts, es-

pecially at short-term horizons. Even though a large number of studies have claimed

M.Rubaszek, et al.

CEJEME 2: 151-167 (2010) 
Forecasting the Polish Zloty with Non-Linear Models

to find success for various versions of fundamentals-based models, sometimes at longer horizons, and over different time periods, the success of these models has not proven to be robust. This exchange rate forecasting puzzle has withstood numerous attempts to resolve it.

The second strand of the literature evolved in a different direction. Instead of testing whether macroeconomic fundamentals convey information that can help explain future exchange rate developments, it investigated whether it is possible to increase the accuracy of exchange rate forecasts in comparison to a random walk model by using advanced, non-linear time series models. Markov-switching (MS) models proposed by Hamilton (1989) were the first group of investigated econometric tools in this kind of studies. Engel and Hamilton (1990) reported that forecasts generated by a univariate two-state regimes MS process tend to be more accurate than those from a random walk model. These results, however, were subsequently rejected for a larger group of currencies, Engel (1994) or longer time samples, Kirikos (2000). As a result, some authors suggest that although MS models fit exchange rate data relatively well, they do not produce superior forecasts to a random walk; e.g. Dacco and Satchell (1999). Artificial neural networks (ANN) constitute the second group of non-linear models used to forecast exchange rates. The findings of 45 journal articles using ANNs for exchange rate forecasting, which are thoroughly surveyed in the book by Yu, Wang, Lai (2007), show that the relative success of ANNs depends on the time sample, the frequency of data and the group of currencies under consideration. Finally, the third group of articles used a smooth transition autoregressive (STAR) model, as proposed by Terasvirta and Anderson (1992). These kind of articles, which followed the studies by Taylor, Peel, Sarno (2001) and Kilian and Taylor (2003), showing that STAR models provide a good description of exchange rate dynamics, are relatively scarce and the results do not point to a significant superiority of STAR models over a random walk; see e.g. Altavilla and De Grauwe (2006). To conclude, even though there is a lot of evidence of non-linearity for exchange rate time series, the out-of-sample forecasts from non-linear econometric models are not consistently more accurate than those from a random walk.

The above discussion is just the very selective review of the most recognized positions from the literature and does not cover the other hundreds of articles on exchange rate forecasting. A more extensive survey by Neely and Sarno (2002) shows that even though the literature on exchange rate forecasting is vast, it is mainly focused on bilateral exchange rates of developed countries. In the case of the Polish zloty the discussion in the literature is scarce. According to our best knowledge there are only two articles that investigate the accuracy of model-based forecasts for the currencies of central and eastern European (CEE) countries; Crespo-Cuaresma and Hlouskova (2005), Ardic, Ergin, Senol (2008). The results of these articles, which are using linear models, show that a random walk model tends to be a very difficult benchmark to beat in the case of the CEE currencies, including the Polish zloty.

This article addresses the relative shortage of the empirical work for the Polish zloty in 
the literature on exchange rate forecasting. We do this by testing whether non-linear time series models are able to generate forecasts for the nominal exchange rate of the Polish zloty that would be more accurate than forecasts from a random walk model. In particular, we analyze the set of competing models consisting of a random walk, fractional random walk, several Markov-switching type models and two variants of ANNs. We test their performance for the bilateral exchange rates of the Polish zloty against the euro, the US dollar, the British pound, the Swiss frank and the Czech koruna. These five bilateral exchange rates were chosen since they are the most important currencies for Polish firms and households.

The article is structured as follows. Section 2 describes the competing models used in our study. Section 3 relates to the issues of the data used in the analysis. Section 4 presents the out-of-sample forecast evaluation results. Section 5 concludes.

\section{Competing models}

\subsection{Random walk}

The benchmark, random walk (RW) model, assumes that variable $y_{t}$ is governed by the unit root process of the form $y_{t}=y_{t-1}+\varepsilon_{t}$, where $\varepsilon_{t} \sim N I D\left(0, \sigma^{2}\right)$ is the random term. In our study $y_{t}$ refers to the log of the exchange rate level. The $h$-step ahead forecast $y_{T+h}$ equals to $y_{T+h}=y_{T}$, where $y_{T}$ is the last available value of the dependent variable in the sample of length $T$.

\subsection{Fractional random walk}

A fractional random walk (FRW) model assumes that variable $y_{t}$ is governed by the fractionally integrated process of the form $(1-L)^{d} y_{t}=\varepsilon_{t}$, where $d$ denotes the integration (differencing) parameter and $\varepsilon_{t} \sim N I D\left(0, \sigma^{2}\right)$ is the random term. For $d \in[0,1]$ the process exhibits so called long-memory or long-range dependency between observations, being covariance-stationary for $d<0.5$ and still mean-reverting for $d<1$. Note that in the case of a random walk model the integration parameter is equal to unity, $d=1$. The fractional differencing operator can be written as $(1-L)^{d}=\sum_{j=0}^{+\infty} \frac{\Gamma(j-d)}{\Gamma(j+1) \Gamma(-d)} L^{j}$, where $\Gamma(\cdot)$ is the gamma function. In our study it is estimated using a frequency domain based method, in particular we apply the Geweke and Porter-Hudak (1993) algorithm of the log periodogram regression. The fractional differencing parameter $d$ is estimated with the gph. $m$ Matlab code developed by Kanzler (1998).

As in the previous case, $y_{t}$ refers to the log of the exchange rate level. The $h$-step ahead forecast $y_{T+h}$ form a fractional random walk model is calculated by using an infinite order moving average (MA) representation of the process. This involves

M.Rubaszek, et al.

CEJEME 2: 151-167 (2010) 
Forecasting the Polish Zloty with Non-Linear Models

computation of the MA coefficients, which are given by the coefficients of the inverse fractional difference operator.

\subsection{Markov-switching models}

The Markov-switching model assumes the existence of an unobserved state variable, $S_{t}$, which in each period takes an integer value form the set $\{1,2, \ldots, K\}$. This state variable characterizes the "state" or "regime" that the process was in at date $t$. When $S_{t}=s$ the dependent variable $y_{t}$ is equal to $y_{t}=X_{t}^{\prime} \beta_{s}+\varepsilon_{t}$, where $\varepsilon_{t} \sim N I D\left(0, \sigma_{s}^{2}\right)$ is the random term, $X_{t}$ is a vector of explanatory variables, $\beta_{s}$ is a vector of model parameters at state $s$, and $t=1,2, \ldots, T$.

The state variable $S_{t}$ is assumed to be the $K$-state Markov chain with probabilities $p_{i j}$, where $i, j=1,2, \ldots, K$. The transition probability $p_{i j}$ describes the probability that state $i$ will be followed by state $j, p_{i j}=P\left(S_{t}=j \mid S_{t-1}=i\right)$. If we define the transition matrix of the process as $P=\left[p_{i j}\right]$ and the probability of the state variable $S_{t}$ being in state $s, \lambda_{t s}=P\left(S_{t}=s\right)$, then the law of motion for the vector $\lambda_{t}=\left[\lambda_{t 1} \lambda_{t 2} \ldots \lambda_{t K}\right]^{\prime}$ is given by $\lambda_{t}=P \lambda_{t-1}$.

The standard method of estimation of the unknown parameters $\beta_{s}, \sigma_{s}^{2}$ and $P$ of the MS model is to maximize the likelihood function calculated with an expectationmaximization algorithm. We use the $M S_{-}$Regress Matlab Toolbox, developed by Perlin (2007), to estimate the parameters of the MS models. Next, the model can be applied to forecasting in two steps. The optimal forecast for $\lambda_{T+h}$ is calculated as $\lambda_{T+h}=P^{h} \lambda_{T}$, and the resulting $h$-step ahead forecast for $y_{T+h}$ is equal to $y_{T+h}=\sum_{s=1}^{K} \lambda_{T+h, s} X_{T+h}^{\prime} \beta_{s}$ (see Hamilton 1994, pp. 694-695). In the case of autoregressive models, forecasts are computed recursively; see Krolizg (2000) for an extended discussion.

In this study we investigate three specifications of the MS model for the logarithmic growth rate of the nominal exchange rate $\left(y_{t}=\Delta e_{t}\right)$. The first two specifications are standard and comparable to those met in the literature; see Engel and Hamilton (1990), Engel (1994). In particular, the set of explanatory variables is limited to a constant. As a result, the growth rate of the exchange rate is assumed to be generated by the process $\triangle e_{t}=\mu_{s}+\varepsilon_{t}, \varepsilon_{t} \sim \operatorname{NID}\left(0, \sigma_{s}^{2}\right)$, where $s \in\{1,2\}$ for the MS(2) model and $s \in\{1,2,3\}$ for the $\operatorname{MS}(3)$ model. The third specification is relatively nonstandard and motivated by the literature on trading strategies on the foreign exchange market, which was initialized by Frankel and Froot (1990) and extensively elaborated on in the book by De Grauwe and Grimaldi (2006). In particular, we assume that the first state describes the market dominated by fundamentalists, which means that the nominal exchange rate is reverting to its fundamental value $\bar{e}$ calculated with the Hodrick-Prescott filter: $\triangle e_{t}=\delta\left(e_{t-1}-\bar{e}_{t-1}\right)+\varepsilon_{t}$, where $\varepsilon_{t} \sim N I D\left(0, \sigma_{1}^{2}\right)$. The smoothing parameter $\lambda$ in the Hodrick-Prescott filter is equal to 46,914,217, which in case of weekly time series corresponds to the "standard" value of 1600 set for 
quarterly time series; see Maravall and del Rio (2001). The second state represents the market dominated by chartists extrapolating trends: $\Delta e_{t}=\rho \triangle e_{t-1}+\varepsilon_{t}$, where $\varepsilon_{t} \sim N I D\left(0, \sigma_{2}^{2}\right)$. We call this specification MS(TS).

\subsection{Artificial neural networks}

Artificial neural networks (ANNs) constitute a parameterized family of regression models which can be utilized for fitting an arbitrary functional form, $f: x \mapsto y$, where the observable input variable $x \in \mathbb{R}^{m}$ and the observable output variable $y \in \mathbb{R}^{n}$. Since in our study we apply ANNs for exchange rate forecasting, the output variable $y$ is the $\log$ of the nominal exchange rate level, which means that $n=1$. The input variable $x$, which represents a set of regressors that are chosen for forecasting $y$, consists of lagged values of $y$ up to lag $K$.

The structure of an ANN with $p$ hidden layers and with $q$ neurons in each hidden layer is represented by Figure 1. The value of neuron $i$ in the first hidden layer is given by $z_{1 i}=g\left(x^{\prime} w_{1 i}+v_{1 i}\right)$, where $g$ is an activation function, $x=\left[x_{1} x_{2} \ldots x_{m}\right]^{\prime}$ is a vector of the input variables, $w_{1 i}=\left[w_{1 i, 1} w_{1 i, 2} \ldots w_{1 i, m}\right]^{\prime}$ is a vector of weights, $v_{1 i}$ is a constant and $i=1,2, \ldots, q$. Subsequently, the value of neurons in the other hidden layers equals to $z_{j i}=g\left(z_{j-1}^{\prime} w_{j i}+v_{j i}\right)$, where $z_{j}=\left[z_{j, 1} z_{j, 2} \ldots z_{j, q}\right]^{\prime}$ is a vector of neurons in the $j$-th hidden layer, $w_{j i}=\left[w_{j i, 1} w_{j i, 2} \ldots w_{j i, q}\right]^{\prime}$ is a vector of weights, $v_{j i}$ is a constant and $j=2, \ldots, p$. Finally, the fitted output variable $\widehat{y}$ is a linear combination of neurons from the $p$-th hidden layer $\widehat{y}=z_{p}^{\prime} w+v$, where $w=\left[w_{1} w_{2} \ldots w_{q}\right]^{\prime}$ is a vector of weights and $v$ is a constant.

Figure 1: The architecture of an artificial neural network

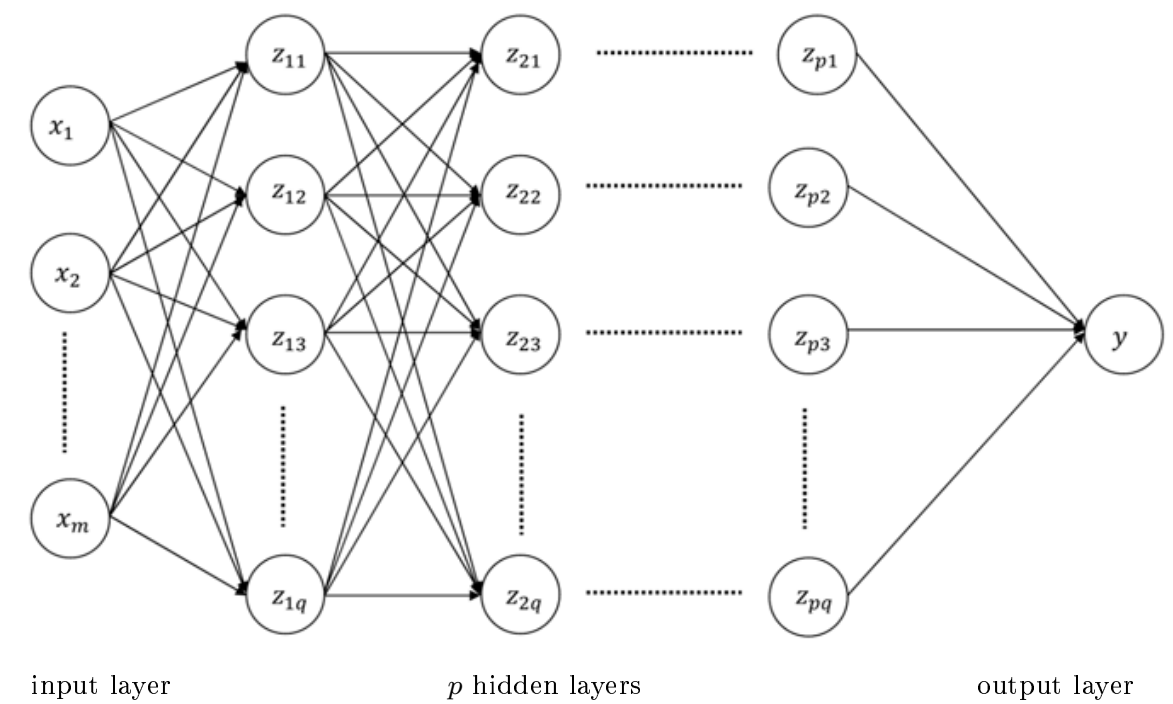

M.Rubaszek, et al.

CEJEME 2: 151-167 (2010) 
Forecasting the Polish Zloty with Non-Linear Models

The number of hidden layers $p$, the number of neurons in each layer $q$, the shape of the activation function $g$ as well as the set of input variables $x$, governed by the maximum lag parameter $K$, are chosen on the basis of numerical experiments. We were testing many ANNs, including a very small one $(p=1$ and $q=1)$ and a very large one $(p=10$ and $q=10)$. In the results section we present the forecasting performance of two ANNs: the small one for which $p=1, q=2$ and $K=3$ (ANN-S), and the large one for which $p=3, q=3$ and $K=10$ (ANN-L). In both cases the activation function $g$ is assumed to be of a hyperbolic tangent form, which generally reflects choices made in the articles reviewed by Yu, Wang, Lai (2007).

The unknown parameters of ANNs, given by vectors $w_{j i}$ and $w$ and scalars $v_{j i}$ and $v$ for $j=1,2, \ldots, p$ and $i=1,2, \ldots, q$, are computed by minimizing the in-sample sum of squared errors $\widehat{e}=y-\widehat{y}$. For that purpose we use the backpropagation technique; see Rumelhart, Hinton, Williams (1986) with the Levenberg-Marquardt algorithm. The $h$-step ahead forecasts $y_{T+h}$ are calculated recursively according to the formula $y_{T+h}=f\left(y_{T+h-1}, y_{T+h-2}, \ldots, y_{T+h-K}\right)$.

\section{The data}

We test the models introduced in the previous section on the basis of weekly, end-ofperiod data for the nominal exchange rate of the Polish zloty against the euro, the US dollar, the British pound, the Swiss frank and the Czech koruna. These five bilateral exchange rates were chosen since they are the most important currencies for Polish firms and households. The models are estimated and used for forecasting on the set of the recursive samples, each starting in the first week of 1999 (1999:w1) and ending in one of the weeks from the period 2004:w1-2009:w52. For instance, the first set of models is estimated with the use of the time series covering the period 1999:w12004:w1 (261 weekly observations) and used for out-of-sample forecasting for 52 weeks starting in the second week of 2004. The second sample for estimation covered one weekly observation more (262 weekly observations). Subsequently, the last recursive sample used covered the period 1999:w1-2009:w52 (573 weekly observations). As a result, each model for each of the five analysed exchange rates is estimated and used for forecasting 313 times. Finally, in each forecasting round we control for the outliers in the forecasts. Namely, we allow the forecasted paths of the exchange rates to vary within the $\pm 5 \%$ band in reference to the last observation. The results of the recursive forecasts for the log of the EUR/PLN are presented in Figure 2. 
Figure 2: EUR/PLN exchange rate out-of-sample forecasts

RW

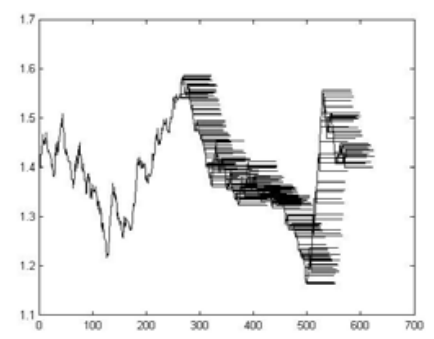

$\mathrm{MS}(2)$

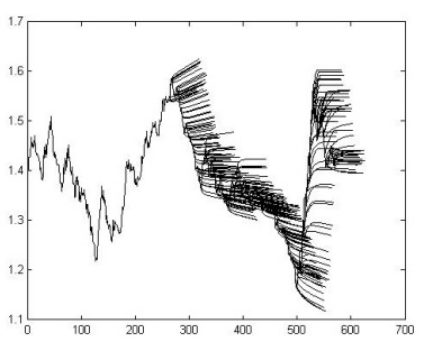

MS(TS)

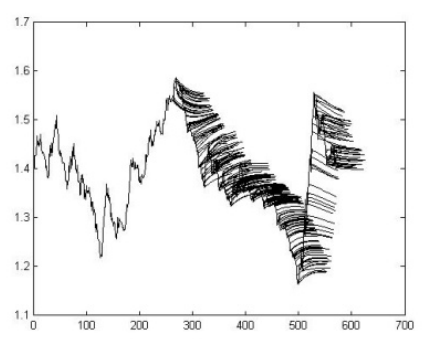

FRW

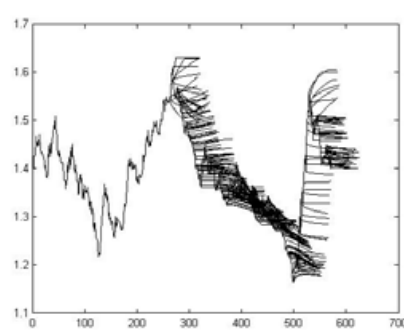

$\mathrm{MS}(3)$

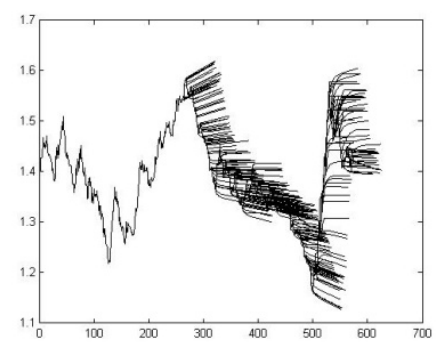

ANN-L

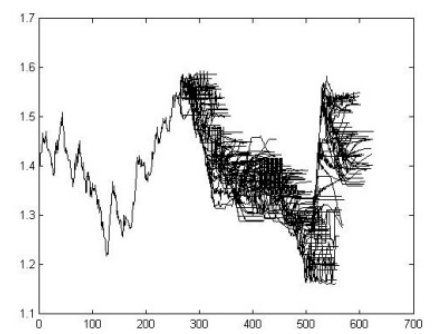

ANN-S

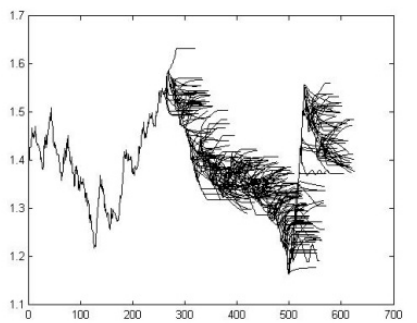

Notes: log levels of EUR/PLN. RW - random walk, FRW - fractional random walk, MS(2) - Markov switching with two regimes, MS(3) - Markov switching with three regimes, MS(TS) - Markov switching with two trading strategy regimes, ANN-L - large artificial neural network, ANN-S - small artificial neural network.

M.Rubaszek, et al. 
Forecasting the Polish Zloty with Non-Linear Models

\section{Out-of-sample forecasts comparison}

The main focus of this study is to check whether out-of-sample forecasts of the Polish zloty exchange rate from the models described in section 2 are more accurate than forecasts from a simple random walk. For that reason we compare the standard measures of forecast accuracy: mean forecast errors (MFEs) and root mean squared forecast errors (RMSFEs). Subsequently, we test the null of forecast unbiasedness as well as the null of equal forecast accuracy of a given model and a random walk. To test the null of forecast unbiasedness we use the $p$-value of the coefficient of the forecast errors regression on a constant. In other words, we test whether the MFE is significantly different from zero. To correct for heteroskedasticity and autocorrelation we use the $\mathrm{HAC}$ covariance matrix estimates obtained via the modified Bartlett kernel in line with Newey and West (1987), where the truncation lag is set automatically as proposed by Newey and West (1994). In order to test the null of equal forecast accuracy we use the Harvey, Leybourne, Newbold (1997) modification of the Diebold and Mariano (1995) test, with the long-run variance estimated via the modified Bartlett kernel, where the truncation lag is set to $h-1$. We also carry out pair-wise forecast encompassing tests, where random walk forecasts are again treated as a benchmark. One forecast encompasses its competitor in the sense that the competitor forecast contains no useful information not present in the superior forecast. The performed pair-wise forecast encompassing tests are based on the auxiliary regressions of the type $y_{t+h}=\beta_{0}+\beta_{M} F_{t+h}^{M}+\beta_{R W} F_{t+h}^{R W}+\varepsilon_{t+h}$, where $y_{t+h}$ denotes the observed exchange rate, $F_{t+h}^{M}$ is the forecast from model $\mathrm{M}$, and $F_{t+h}^{R W}$ is the random walk forecast. If and only if the $\beta_{M}$ coefficient is significantly different from zero the forecast generated by model $\mathrm{M}$ is said to encompass the forecast generated by a random walk. To correct for heteroskedasticity and autocorrelation we use the HAC covariance matrix estimates obtained via the modified Bartlett kernel in line with Newey and West (1987), where the truncation lag is set automatically as proposed by Newey and West (1994). For references on this type of forecast encompassing test; see Clements and Harvey (2006) or Romer and Romer (2000). The forecasting horizon ranges from one to 52 weeks, and in particular the presentation of the results focuses on horizons of $1,4,8,12,26$ and 52 weeks.

The forecasts are evaluated with the recursive data from the period 2004:w2-2009:w52. For one-week ahead forecasts we use all 312 weekly observations from that period. Generally, in the case of $h$-step ahead forecasts, the evaluation sample is truncated of the first $h-1$ observations, for which forecasts are not available. This means that 52-weeks ahead forecasts are compared with 261 observations from the period 2004:w53-2009:w52. In the following part of this section we report the results of the unbiasedness, the equal forecast accuracy and the forecast encompassing tests.

Table 1 reports the results of the forecast unbiasedness tests. The main conclusion which builds on these results is that most of the obtained forecasts are unbiased with some exceptions, relating mostly to the GBP/PLN and USD/PLN exchange rates. 
M. Rubaszek, P. Skrzypczyński, G. Koloch

Table 1: MFEs and the forecast unbiasedness test

\begin{tabular}{|c|c|c|c|c|c|c|c|}
\hline \multicolumn{8}{|c|}{ EUR/PLN } \\
\hline$h$ & RW & FRW & $\mathrm{MS}(2)$ & $\operatorname{MS}(3)$ & $\mathrm{MS}(\mathrm{TS})$ & ANN-L & ANN-S \\
\hline 1 & -0.0004 & 0.0005 & -0.0006 & -0.0009 & -0.0008 & -0.0017 & -0.0010 \\
\hline 4 & -0.0016 & 0.0013 & -0.0025 & -0.0034 & -0.0033 & -0.0042 & -0.0032 \\
\hline 8 & -0.0036 & 0.0014 & -0.0054 & -0.0062 & -0.0068 & -0.0081 & -0.0063 \\
\hline 12 & -0.0054 & 0.0015 & -0.0073 & -0.0082 & -0.0098 & -0.0100 & -0.0087 \\
\hline 26 & -0.0112 & 0.0012 & -0.0115 & -0.0132 & -0.0189 & -0.0187 & -0.0147 \\
\hline 52 & -0.0094 & 0.0113 & -0.0025 & -0.0051 & -0.0224 & -0.0193 & -0.0139 \\
\hline \multicolumn{8}{|c|}{ USD/PLN } \\
\hline$h$ & RW & FRW & $\operatorname{MS}(2)$ & $\operatorname{MS}(3)$ & $\mathrm{MS}(\mathrm{TS})$ & ANN-L & ANN-S \\
\hline 1 & -0.0008 & -0.0014 & $-0.0024^{*}$ & $-0.0024^{*}$ & -0.0023 & $-0.0059^{* * *}$ & $-0.0040^{* * *}$ \\
\hline 4 & -0.0034 & -0.0040 & -0.0071 & -0.0072 & $-0.0089^{*}$ & $-0.0109^{*}$ & $-0.0121 * *$ \\
\hline 8 & -0.0077 & -0.0079 & -0.0106 & -0.0106 & $-0.0175^{*}$ & $-0.0166^{*}$ & $-0.0186^{*}$ \\
\hline 12 & -0.0119 & -0.0117 & -0.0138 & -0.0137 & $-0.0243 * *$ & $-0.0209^{*}$ & $-0.0233^{*}$ \\
\hline 26 & -0.0264 & -0.0259 & -0.0233 & -0.0228 & $-0.0390^{*}$ & -0.0356 & $-0.0392 *$ \\
\hline 52 & -0.0320 & -0.0267 & -0.0152 & -0.0145 & -0.0298 & -0.0425 & $-0.0461^{*}$ \\
\hline \multicolumn{8}{|c|}{ GBP/PLN } \\
\hline$h$ & RW & FRW & $\mathrm{MS}(2)$ & $\operatorname{MS}(3)$ & MS(TS) & ANN-L & ANN-S \\
\hline 1 & -0.0012 & 0.0002 & -0.0013 & -0.0014 & -0.0016 & -0.0018 & -0.0010 \\
\hline 4 & -0.0050 & -0.0002 & -0.0052 & -0.0057 & $-0.0063^{*}$ & $-0.0076^{*}$ & -0.0055 \\
\hline 8 & $-0.0107^{*}$ & -0.0024 & $-0.0107^{*}$ & $-0.0115^{*}$ & $-0.0133^{* *}$ & $-0.0154^{* *}$ & $-0.0116^{*}$ \\
\hline 12 & $-0.0166^{* *}$ & -0.0059 & $-0.0164 * *$ & $-0.0174^{* *}$ & $-0.0205^{* * *}$ & $-0.0227 * * *$ & $-0.0181 * *$ \\
\hline 26 & $-0.0362^{* * *}$ & -0.0194 & $-0.0344 * * *$ & $-0.0345 * * *$ & $-0.0431^{* * *}$ & $-0.0432^{* * *}$ & $-0.0401 * * *$ \\
\hline 52 & $-0.0636^{* * *}$ & $-0.0372 * *$ & $-0.0577^{* * *}$ & $-0.0563 * * *$ & $-0.0747 * * *$ & $-0.0728^{* * *}$ & $-0.0721^{* * *}$ \\
\hline \multicolumn{8}{|c|}{ CHF/PLN } \\
\hline$h$ & RW & FRW & $\mathrm{MS}(2)$ & $\operatorname{MS}(3)$ & MS(TS) & ANN-L & ANN-S \\
\hline 1 & -0.0003 & -0.0005 & -0.0009 & -0.0014 & -0.0012 & -0.0005 & -0.0011 \\
\hline 4 & -0.0011 & -0.0013 & -0.0036 & -0.0047 & -0.0039 & -0.0031 & -0.0033 \\
\hline 8 & -0.0026 & -0.0024 & -0.0055 & -0.0067 & -0.0078 & -0.0059 & -0.0071 \\
\hline 12 & -0.0040 & -0.0032 & -0.0067 & -0.0081 & -0.0111 & -0.0079 & -0.0100 \\
\hline 26 & -0.0088 & -0.0055 & -0.0109 & -0.0125 & -0.0220 & -0.0155 & -0.0176 \\
\hline 52 & -0.0050 & 0.0043 & -0.0010 & -0.0032 & -0.0221 & -0.0138 & -0.0174 \\
\hline \multicolumn{8}{|c|}{ CZK/PLN } \\
\hline$h$ & RW & FRW & $\mathrm{MS}(2)$ & $\operatorname{MS}(3)$ & MS(TS) & ANN-L & ANN-S \\
\hline 1 & 0.0003 & 0.0007 & 0.0000 & -0.0002 & -0.0003 & 0.0009 & 0.0011 \\
\hline 4 & 0.0014 & 0.0027 & -0.0002 & -0.0007 & -0.0010 & 0.0025 & 0.0031 \\
\hline 8 & 0.0026 & 0.0047 & -0.0009 & -0.0017 & -0.0015 & 0.0037 & 0.0044 \\
\hline 12 & 0.0040 & 0.0068 & -0.0016 & -0.0024 & -0.0015 & 0.0053 & 0.0052 \\
\hline 26 & 0.0098 & $0.0150^{*}$ & -0.0026 & -0.0035 & 0.0000 & 0.0111 & 0.0084 \\
\hline 52 & $0.0283^{* * *}$ & $0.0393 * * *$ & 0.0055 & 0.0040 & 0.0066 & $0.0288 * *$ & $0.0235^{* *}$ \\
\hline
\end{tabular}

Notes: bold figures indicate minimal absolute value of the MFE for a given forecast horizon $h$. A positive MFE indicates that on average forecasts are below the actual values. Symbols ***, ${ }^{* *}$ and * indicate the rejection of the null that the MFE is equal to zero at $1 \%, 5 \%$ and $10 \%$ significance levels, respectively.

M.Rubaszek, et al.

CEJEME 2: 151-167 (2010) 
Forecasting the Polish Zloty with Non-Linear Models

In particular, for the GBP/PLN exchange rate all models but a fractional random walk generate biased forecasts, whereas for the USD/PLN exchange rate MS(TS) model and ANNs tend to produce forecasts with MFEs significantly different from zero. As regards the comparison of the absolute values of the MFEs, FRW, RW and MS(2) tend to outperform the other analysed models (see Table 1).

Table 2 reports the results of the equal forecast accuracy test. They indicate that a random walk model is a relatively strong benchmark. In fact the only case where, at the $1 \%$ significance level, the RMSFE of a given model turned out to be significantly lower than that of a random walk is the one for FRW model forecasts of the CHF/PLN exchange rate at the 52-weeks ahead horizon. Assuming the $10 \%$ significance level we find that FRW model also outperforms a random walk in forecasting the GBP/PLN exchange rate at the 52-weeks ahead horizon, as does MS(TS) model in case of the USD/PLN exchange rate at the same horizon. There are also other cases where the analysed models, including FRW, the Markov-switching type ones and ANNs, generate lower RMSFEs than a random walk, however the resulting differences in the obtained errors turn out to be not significantly different from zero. On the other hand there are many cases where a random walk model significantly outperforms the other methods in forecasting the PLN exchange rate. This is most evident in the case of ANNs. Especially, ANN-L tends to perform worse than a random walk at roughly all considered horizons in each exchange rate case. The poor performance of ANN-S is most evident in the case of the USD/PLN and GBP/PLN exchange rates. Furthermore, FRW model forecasts are significantly worse than those from a random walk at roughly all horizons for the CZK/PLN exchange rate and in the case of the short-term forecasts of the EUR/PLN and GBP/PLN exchange rates. As regards the Markov-switching models, we find that MS(TS) model is generally outperformed by a random walk in short-term forecasting of the USD/PLN and the CHF/PLN exchange rates. In all cases relating to $\mathrm{MS}(2)$ and $\mathrm{MS}(3)$ models we find that the null of equal forecast accuracy between these models and a random walk can not be rejected.

Table 3 shows the results of the forecast encompassing tests. The general picture that emerges is that only in the case of the EUR/PLN and CHF/PLN exchange rates Markov-switching models forecasts carry a richer informational content than random walk forecasts. In particular, for these two currencies forecasts from the MS(3) model are found to encompass random walk forecasts at horizons up to 12-weeks ahead. The same refers to MS(2) model forecasts, though only in the EUR/PLN exchange rate case. Some evidence in favour of the MS(3) model is also found in case of the $\mathrm{CZK} / \mathrm{PLN}$ exchange rate forecasts, however this refers only to 4 - and 12-weeks ahead forecasts. There are also other cases where the analysed models turn out to encompass a random walk in forecasting the Polish zloty, however these are enumerative examples referring mainly to a FRW and the MS(2) model, and even to a smaller degree to the MS(TS) model. Finally, we find that the ANNs are not able to generate forecasts of the Polish zloty with richer informational content than naive no change predictions. To summarize, the following conclusions are in place. First, in our exchange rate 
M. Rubaszek, P. Skrzypczyński, G. Koloch

Table 2: RMSFEs and the equal forecast accuracy test

\begin{tabular}{|c|c|c|c|c|c|c|c|}
\hline \multicolumn{8}{|c|}{ EUR/PLN } \\
\hline$h$ & RW & FRW & $\mathrm{MS}(2)$ & $\operatorname{MS}(3)$ & $\mathrm{MS}(\mathrm{TS})$ & ANN-L & ANN-S \\
\hline 1 & 0.0157 & $1.02^{* *}$ & 1.00 & 0.98 & 0.99 & $1.14^{* * *}$ & $1.05^{*}$ \\
\hline 4 & 0.0291 & $1.03^{*}$ & 0.99 & 0.97 & $1.02 *$ & $1.15^{* * *}$ & $1.07 *$ \\
\hline 8 & 0.0447 & 1.02 & 1.00 & 0.98 & 1.02 & $1.13^{* * *}$ & 1.05 \\
\hline 12 & 0.0574 & 1.01 & 1.00 & 0.99 & 1.02 & $1.09 *$ & 1.04 \\
\hline 26 & 0.0979 & 0.99 & 1.05 & 1.04 & 1.00 & 1.03 & 0.99 \\
\hline 52 & 0.1265 & 1.01 & 1.08 & 1.06 & 1.00 & 0.97 & 0.94 \\
\hline \multicolumn{8}{|c|}{$\frac{1}{\mathrm{USD} / \mathrm{PLN}}$} \\
\hline$h$ & RW & FRW & $\operatorname{MS}(2)$ & $\operatorname{MS}(3)$ & $\mathrm{MS}(\mathrm{TS})$ & ANN-L & ANN-S \\
\hline 1 & 0.0229 & 1.04 & 1.04 & 1.03 & $1.04^{*}$ & $1.26^{* * *}$ & $1.08^{* * *}$ \\
\hline 4 & 0.0462 & 1.00 & 1.05 & 1.04 & $1.04^{* *}$ & $1.21^{* * *}$ & $1.14^{* * *}$ \\
\hline 8 & 0.0703 & 0.97 & 1.03 & 1.03 & $1.05^{*}$ & $1.13^{* * *}$ & $1.17^{* * *}$ \\
\hline 12 & 0.0892 & 0.96 & 1.02 & 1.02 & 1.05 & $1.08^{* * *}$ & $1.13^{* * *}$ \\
\hline 26 & 0.1547 & 0.96 & 1.04 & 1.04 & 1.00 & $1.05^{* *}$ & $1.10^{* * *}$ \\
\hline 52 & 0.1955 & 0.97 & 1.05 & 1.05 & $0.94 *$ & 1.04 & 1.07 \\
\hline \multicolumn{8}{|c|}{ GBP/PLN } \\
\hline$h$ & RW & FRW & $\operatorname{MS}(2)$ & $\operatorname{MS}(3)$ & MS(TS) & ANN-L & ANN-S \\
\hline 1 & 0.0177 & $1.05^{* * *}$ & 1.00 & 1.01 & 1.00 & $1.27^{* * *}$ & $1.12^{* * *}$ \\
\hline 4 & 0.0342 & $1.09^{* * *}$ & 1.01 & 1.04 & 1.01 & $1.26^{* * *}$ & $1.14^{* * *}$ \\
\hline 8 & 0.0490 & $1.08^{* *}$ & 1.02 & 1.06 & 1.01 & $1.19 * * *$ & $1.14^{* *}$ \\
\hline 12 & 0.0591 & 1.06 & 1.01 & 1.06 & 1.01 & $1.13^{* *}$ & $1.13^{* *}$ \\
\hline 26 & 0.0984 & 0.96 & 1.03 & 1.04 & 0.99 & 1.09 & 1.08 \\
\hline 52 & 0.1371 & $0.84^{*}$ & 1.04 & 1.04 & 0.96 & 1.07 & 1.08 \\
\hline \multicolumn{8}{|c|}{$\mathrm{CHF} / \mathrm{PLN}$} \\
\hline$h$ & RW & FRW & $\operatorname{MS}(2)$ & $\operatorname{MS}(3)$ & MS(TS) & ANN-L & ANN-S \\
\hline 1 & 0.0189 & 1.00 & 1.01 & 1.00 & 1.02 & $1.09 * * *$ & 1.03 \\
\hline 4 & 0.0352 & 1.00 & 1.02 & 0.98 & $1.03^{* *}$ & $1.14^{* * *}$ & 1.02 \\
\hline 8 & 0.0532 & 1.00 & 1.00 & 0.99 & $1.03^{*}$ & $1.11^{* * *}$ & 1.02 \\
\hline 12 & 0.0682 & 0.98 & 1.01 & 1.00 & 1.04 & $1.09 * * *$ & 1.02 \\
\hline 26 & 0.1135 & 0.97 & 1.04 & 1.04 & 1.02 & 1.01 & 0.99 \\
\hline 52 & 0.1498 & $0.95 * * *$ & 1.06 & 1.06 & 1.05 & 0.99 & 0.97 \\
\hline \multicolumn{8}{|c|}{ CZK/PLN } \\
\hline$h$ & RW & FRW & $\operatorname{MS}(2)$ & $\operatorname{MS}(3)$ & MS(TS) & ANN-L & ANN-S \\
\hline 1 & 0.0128 & 1.01 & 1.01 & 1.00 & 0.99 & $1.29^{* * *}$ & $1.10^{* * *}$ \\
\hline 4 & 0.0241 & $1.04 *$ & 1.01 & 1.00 & 1.00 & $1.26^{* * *}$ & $1.14^{* *}$ \\
\hline 8 & 0.0319 & $1.05^{* *}$ & 1.00 & 1.00 & 1.01 & $1.22^{* *}$ & 1.17 \\
\hline 12 & 0.0368 & $1.05^{* *}$ & 1.00 & 1.01 & 1.03 & $1.23^{* *}$ & 1.16 \\
\hline 26 & 0.0601 & $1.05^{* *}$ & 1.02 & 1.03 & 1.01 & $1.13^{*}$ & 1.03 \\
\hline 52 & 0.0826 & $1.08^{* *}$ & 1.00 & 1.01 & 1.00 & 1.11 & 1.01 \\
\hline
\end{tabular}

Notes: a RW model RMSFEs are reported in levels while other presented figures are ratios of RMSFE from a given model to the corresponding RMSFE from a RW model. A ratio below unity indicates that the RMSFE for a given model is lower than the corresponding one from a RW model (bold figures). Symbols $* * *,{ }^{*}$ and $*$ indicate the rejection of the null of the HLN-DM test, stating that the given RMSFE is not significantly different from the corresponding RMSFE from a RW model, at $1 \%, 5 \%$ and $10 \%$ significance levels, respectively.

M.Rubaszek, et al.

162

CEJEME 2: 151-167 (2010) 
Forecasting the Polish Zloty with Non-Linear Models

Table 3: Forecast encompassing test

\begin{tabular}{|c|c|c|c|c|c|c|c|c|c|c|c|c|}
\hline \multicolumn{13}{|c|}{ EUR/PLN } \\
\hline & \multicolumn{2}{|c|}{ FRW } & \multicolumn{2}{|c|}{ MS (2) } & \multicolumn{2}{|c|}{ MS $(3)$} & \multicolumn{2}{|c|}{ MS(TS) } & \multicolumn{2}{|c|}{ ANN-L } & \multicolumn{2}{|c|}{ ANN-S } \\
\hline & $\beta_{M}$ & $\beta_{R W}$ & $\beta_{M}$ & $\beta_{R W}$ & $\beta_{M}$ & $\beta_{R W}$ & $\beta_{M}$ & $\beta_{R W}$ & $\beta_{M}$ & $\beta_{R W}$ & $\beta_{M}$ & $\beta_{R W}$ \\
\hline 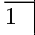 & 0.04 & $0.94^{* * *}$ & $1.02^{* *}$ & -0.05 & $1.10^{* *}$ & -0.13 & $0.75^{*}$ & 0.24 & -0.05 & $1.03^{* * *}$ & $* 0.05$ & $0.94^{* * *}$ \\
\hline & 0.15 & $0.79^{* * *}$ & $1.14^{* *}$ & -0.25 & $1.16^{* * *}$ & ${ }^{*}-0.27$ & $-1.35 *$ & $2.26^{* * *}$ & 0.15 & $1.08 * * *$ & $*-0.29$ & $1.21 * * *$ \\
\hline & 0.28 & $0.56^{* *}$ & $1.25^{* *}$ & -0.49 & $1.39^{* *}$ & -0.63 & $-2.12^{* *}$ & & -0.11 & $0.95^{* * *}$ & $*-0.32^{* *}$ & \\
\hline & $0.61 * *$ & 0.13 & $1.56^{* *}$ & -0.95 & $1.68^{* *}$ & -1.05 & $-2.28 * *$ & 2.91 & -0.14 & $0.87 * * *$ & *- $-0.35^{* *}$ & $1.05^{* * *}$ \\
\hline & $1.39^{* * *}$ & $-1.12^{* *}$ & $2.04^{* * *}$ & $-1.94^{* * *}$ & $* 1.97^{* * *}$ & $-1.84 * * *$ & $-1.45^{*}$ & $1.68^{* * *}$ & -0.26 & $0.58 * * *$ & *- $-0.35^{* *}$ & $0.64^{* * *}$ \\
\hline 2 & $1.71^{* * *}$ & $-1.92^{* * *}$ & $1.53^{* * *}$ & $-1.83^{* * *}$ & ${ }^{*} 2.15^{* * * *}$ & $-2.50 * * *$ & -0.40 & 0.31 & 0.19 & -0.24 & 0.10 & -0.15 \\
\hline \multicolumn{13}{|c|}{ USD/PLN } \\
\hline & \multicolumn{2}{|c|}{ FRW } & \multicolumn{2}{|c|}{ MS (2) } & \multicolumn{2}{|c|}{$\mathrm{MS}(3)$} & \multicolumn{2}{|c|}{ MS(TS) } & \multicolumn{2}{|c|}{ ANN-L } & \multicolumn{2}{|c|}{ ANN-S } \\
\hline & $\beta_{M}$ & $\beta_{R W}$ & $\beta_{M}$ & $\beta_{R W}$ & $\beta_{M}$ & $\beta_{R W}$ & $\beta_{M}$ & $\beta_{R W}$ & $\beta_{M}$ & $\beta_{R W}$ & $\beta_{M}$ & $\beta_{R W}$ \\
\hline & 0.06 & $0.92^{* * *}$ & 0.04 & $0.95^{* * *}$ & 0.22 & $0.76^{* * *}$ & -1.07 & $2.06^{* * *}$ & -0.09 & $1.08^{* * *}$ & *0.03 & $0.95^{* * *}$ \\
\hline & 0.47 & 0.47 & 0.22 & 0.7 & 0.28 & $0.66^{*}$ & & & -0.16 & $1.10^{* * *}$ & & 1.0 \\
\hline & $0.80 * *$ & 0.09 & 0.42 & 0.4 & 0.50 & 0.36 & & & 0.12 & $0.98 * * *$ & & \\
\hline & $0.98^{*}$ & -0.17 & 0.68 & 0.0 & 0.72 & 0.04 & -0.45 & & 0.01 & $0.79 * * *$ & & $1.1^{\prime} \quad$ \\
\hline & $1.00 *$ & -0.54 & 0.33 & 0.0 & 0.39 & 0.00 & -0. & 0. & -0.35 & $0.76^{* *}$ & $-0.97^{* *}$ & 1.3 \\
\hline 2 & -0.87 & 0.93 & 0.73 & 0.69 & 0.15 & -0.05 & $2.27^{* * *}$ & & -0.45 & $0.57^{*}$ & $-1.10^{* *}$ & \\
\hline \multicolumn{13}{|c|}{ GBP/PLN } \\
\hline & \multicolumn{2}{|c|}{ FRW } & \multicolumn{2}{|c|}{$\operatorname{MS}(2)$} & & $\mathrm{S}(3)$ & MS & (TS) & AN & N-L & $\mathrm{AN}$ & $\mathrm{N}-\mathrm{S}$ \\
\hline & $\beta_{M}$ & $\beta_{R W}$ & $\beta_{M}$ & $\beta_{R W}$ & $\beta_{M}$ & $\beta_{R W}$ & $\beta_{M}$ & $\beta_{R W}$ & $\beta_{M}$ & $\beta_{R W}$ & $\beta_{M}$ & $\beta_{R W}$ \\
\hline & $-0.28^{*}$ & $1.27^{* * *}$ & 0.36 & 0.63 & -0.16 & $1.15^{*}$ & 0.14 & 0.85 & -0.14 & $1.13^{* * *}$ & ${ }^{*}-0.10$ & 1.0 \\
\hline & -0.49 & 1.43 & 0.04 & & -0.3 & 1.2 & -0. & & -0.21 & 1.17 & & \\
\hline & $-0.76^{* * *}$ & 1.6 & -0.4 & $1.33^{* *}$ & -0.6 & 1.5 & 0.4 & 0. & -0.16 & $1.07^{* * *}$ & & \\
\hline 2 & $-0.84^{* * *}$ & ${ }^{*} 1.65^{* *}$ & 0.06 & 0.82 & -0.57 & $1.45^{* * *}$ & 0.4 & 0.4 & 0.03 & $0.86^{* * *}$ & $*-0$. & $0.92^{* * *}$ \\
\hline & $-0.75^{* *}$ & 1.35 & -0.71 & 1.4 & $-0.79^{*}$ & 1.49 & & & -0.23 & 0.9 & & \\
\hline & 1.81 & -1.17 & 1.61 & -1.3 & 0.94 & -0.57 & $2.63^{* * *}$ & -1 & $0.58^{*}$ & $1.03^{\circ}$ & & \\
\hline & & & & & & $\mathrm{CHF}$ & LN & & & & & \\
\hline & & RW & & $\mathrm{S}(2)$ & & $\mathrm{S}(3)$ & MS & (TS) & AN & N-L & AN I & $\mathrm{N}-\mathrm{S}$ \\
\hline & $\beta_{M}$ & $\beta_{R W}$ & $\beta_{M}$ & $\beta_{R W}$ & $\beta_{M}$ & $\beta_{R W}$ & $\beta_{M}$ & $\beta_{R W}$ & $\beta_{M}$ & $\beta_{R W}$ & $\beta_{M}$ & $\beta_{R W}$ \\
\hline & 0.20 & $0.79^{*}$ & 0.44 & & 0.71 & 0.26 & & & $0.24^{*}$ & $0.75^{* * *}$ & $* 0.20$ & \\
\hline & 0.29 & 0.66 & 0.7 & 0.2 & 1.0 & ${ }^{*}-0.18$ & & & -0.0 & 1.00 & & \\
\hline & -0.05 & 0.92 & & -0.4 & & $*-0.77$ & & & 0. & $1.01 * * *$ & & \\
\hline & 0.50 & 0.30 & $1.56^{* *}$ & -0.8 & $1.89^{* *}$ & -1.23 & $-1.60 * *$ & & $-0.29^{*}$ & $1.04^{* * *}$ & & \\
\hline & $1.60^{* *}$ & -1.12 & $2.68^{* * *}$ & -2.5 & 2.6 & $-2.50 * * *$ & -1.2 & & $-0.40^{* *}$ & $0.78^{* * *}$ & & \\
\hline 2 & $2.39 * * *$ & -2.30 & $4.25^{* * *}$ & -4.8 & $3.35^{* * *}$ & $-3.82^{* * *}$ & $-1.29^{* *}$ & 1.3 & $-0.50 * *$ & $0.44^{* *}$ & & 0.40 \\
\hline & & & & & & $\mathrm{CZK} / \mathrm{P}$ & LN & & & & & \\
\hline & $\mathrm{FF}$ & RW & & $\mathrm{S}(2)$ & & $\mathrm{S}(3)$ & MS & (TS) & AN & N-L & $\mathrm{AN}$ & $\mathrm{N}-\mathrm{S}$ \\
\hline & $\beta_{M}$ & $\beta_{R W}$ & $\beta_{M}$ & $\beta_{R W}$ & $\beta_{M}$ & $\beta_{R W}$ & $\beta_{M}$ & $\beta_{R W}$ & $\beta_{M}$ & $\beta_{R W}$ & $\beta_{M}$ & $\beta_{R W}$ \\
\hline & -0.17 & & 0.82 & 0.16 & 0.98 & -0.01 & 0.69 & & -0.0 & & & \\
\hline & -0.5 & & & & & -0.85 & & & & $1.07 * * *$ & & \\
\hline & -0.56 & $1.49^{*}$ & $2.47^{* * *}$ & $-1.70^{*}$ & $2.62^{* * *}$ & $-1.80^{*}$ & -0.30 & 1.2 & -0.09 & $1.01 * * *$ & $*-0$. & $1.06^{* * *}$ \\
\hline & -0.40 & & $2.78^{* * *}$ & $-2.10^{* *}$ & $1.45^{*}$ & -0.62 & -1.05 & & -0.14 & & & \\
\hline & 0.00 & $0.75^{*}$ & 1.99 & -1.51 & $-2.89^{*}$ & $3.92^{* *}$ & $-2.81^{* * *}$ & & -0.23 & $0.95^{* * *}$ & & \\
\hline 52 & 0.30 & 0.01 & $-6.08^{* * *}$ & $* 7.96 * * *$ & $-8.61^{* * *}$ & $10.79^{* *}$ & $-4.54^{* * *}$ & $2.91^{* * *}$ & $-0.79^{*}$ & $0.84^{* * *}$ & $*-0.57^{* *}$ & $0.57^{*}$ \\
\hline
\end{tabular}

Notes: bold figures indicate cases where the coefficient representing a given model forecast is significantly different from zero at $1 \%, 5 \%$ or $10 \%$ significance levels, while the coefficient representing a random walk forecast is insignificant. These cases indicate the evidence of forecast encompassing by a given model in reference to a random walk. Symbols $* * *, * *$ and $*$ indicate the rejection of the null that the given coefficient is equal to zero at $1 \%, 5 \%$ and $10 \%$ significance levels, respectively. 
forecasting contest the Markov-switching models performed somewhat better than the ANNs, which needs further investigation. Second, even though the Markov-switching models were well suited to describe in-sample dynamics of the exchange rates, they were unable to predict the out-of-sample turning points. Third, we were not able to find any monotone relationship between ANNs' forecasting performance and its internal structure, but the general tendency that the small ANN tended to produce smaller out-of-sample errors than the large ANN. The large ANN better described insample exchange rate dynamics. However, it also produced higher volatility forecasts with larger out-of-sample errors. It should be noted, that smaller networks produce less volatile forecasts, but do not extrapolate complex behaviour of the exchange rates. If exchange rate series were to be characterised by highly non-linear, self-repeating patterns, then, since larger networks are capable of capturing these dynamics, one would expect them to produce more accurate forecasts. Our experiment suggests however, that this is not the case. Finally, the main conclusion of our study is that the analysed models were not able to consistently outperform the random walk in forecasting the Polish zloty.

\section{Conclusions}

The literature on exchange rate forecasting indicates that there is no model that is able to consistently outperform a random walk in exchange rate forecasting. Even though a large number of studies have claimed to find success in exchange rate forecasting, this success has not proven to be robust. This exchange rate forecasting puzzle has withstood numerous attempts to resolve it.

This article adds to this extensive literature by analysing whether it is possible to forecast the Polish zloty with linear and non-linear time series models. Even though for some forecast horizons, selected currencies and forecast evaluation measures we found some evidence in favour of a given model in comparison to a random walk, the conclusion drawn from our investigation is that the general belief that exchange rates are difficult to forecast also holds for the Polish zloty. We found that a random walk model tends to explain future movements of the PLN exchange rate against five foreign currencies comparably or even better than the other investigated models. Finally, it is worth to outline that the obtained results are consistent with the market efficiency hypothesis.

\section{Acknowledgement}

The earlier version of this article has been published in the National Bank of Poland Working Paper Series.

M.Rubaszek, et al.

CEJEME 2: 151-167 (2010) 
Forecasting the Polish Zloty with Non-Linear Models

\section{References}

[1] Altavilla C., De Grauwe P., (2006), Forecasting and combining competing models of exchange rate determination, CESifo Working Paper Series 1747, Munich.

[2] Ardic O.P., Ergin O., Senol G.B., (2008), Exchange rate forecasting: Evidence from the emerging central and eastern European economies, MPRA Paper 7505, Munich.

[3] Berkowitz J., Giorgianni L., (2001), Long-horizon exchange rate predictability?, Review of Economics and Statistics 83, 81-91.

[4] Canova F., (1993), Modelling and forecasting exchange rates with a Bayesian time-varying coefficient model, Journal of Economic Dynamics and Control 17, 233-261.

[5] Cheung Y.-W., Chinn M.D., Pascual A.G., (2005), Empirical exchange rate models of the nineties: Are any fit to survive?, Journal of International Money and Finance 24, 1150-1175.

[6] Chinn M.D., Meese R.A., (1995), Banking on currency forecasts: How predictable is change in money?, Journal of International Economics 38, 161-178.

[7] Clements M.P., Harvey D.I., (2006), Forecast encompassing tests and probability forecasts, University of Warwick, Department of Economics Research Paper Series 774, Warwick.

[8] Crespo-Cuaresma J., Hlouskova J., (2005), Beating the random walk in central and eastern Europe, Journal of Forecasting 24, 189-201.

[9] Dacco R., Satchell C., (1999), Why do regime-switching forecast so badly?, Journal of Forecasting 18, 1-16.

[10] De Grauwe P., Grimaldi M., (2006), The Exchange Rate in a Behavioral Finance Framework, Princeton University Press, New Jersey.

[11] Diebold F.X., Mariano R.S., (1995), Comparing predictive accuracy, Journal of Business \& Economic Statistics 13, 134-144.

[12] Engel Ch., (1994), Can the Markov switching model forecast exchange rates?, Journal of International Economics 36, 151-165.

[13] Engel Ch., Hamilton J.D., (1990), Long swings in the dollar: Are they in the data and do markets know it?, American Economic Review 80, 689-713.

[14] Engel Ch., West K., (2005), Exchange rates and fundamentals, Journal of Political Economy 113, 485-517. 
[15] Faust J., Rogers J., Wright J., (2003), Exchange rate forecasting errors we've really made, Journal of International Economics 60, 35-59.

[16] Frankel J.A., Froot K.A., (1990), Chartists, fundamentalists, and trading in the foreign exchange market, American Economic Review 80, 181-185.

[17] Geweke J., Porter-Hudak S., (1983), The estimation and application of longmemory time series models, Journal of Time Series Analysis 4, 221-228.

[18] Hamilton J.D., (1989), A new approach to the economic analysis of nonstationary time series and the business cycle, Econometrica 57, 357-384.

[19] Hamilton J.D., (1990), Analysis of time series subject to changes in regime, Journal of Econometrics 45, 39-70.

[20] Hamilton J.D., (1994), Time Series Analysis, Princeton University Press, New Jersey.

[21] Harvey D., Leybourne S., Newbold P., (1997), Testing the equality of prediction mean squared errors, International Journal of Forecasting 13, 281-291.

[22] Kanzler L., (1998), GPH: MATLAB module to calculate Geweke-Porter-Hudak long memory statistic, Boston College Department of Economics Statistical Software Components T850805, Boston.

[23] Kilian L., (1999), Exchange rates and monetary fundamentals: What do we learn from long-horizon regressions?, Journal of Applied Econometrics 14, 491-510.

[24] Kilian L., Taylor M. P., (2003), Why is it so difficult to beat the random walk forecast of exchange rates?, Journal of International Economics 60, 85-107.

[25] Kirikos D.G., (2000), Forecasting exchange rates out of sample: Random walk vs Markov switching regimes, Applied Economics Letters 7, 133-136.

[26] Krolzig H.M., (2000), Predicting Markov-switching vector autoregressive processes, Nuffield College Economics Discussion Paper 31, Oxford.

[27] Maravall A., del Rio A., (2001), Time aggregation and the Hodrick-Prescott filter, Banco de España Working Papers 0108, Madrid.

[28] Mark N., (1995), Exchange rates and fundamentals: Evidence on long-horizon predictability, American Economic Review 85, 201-218.

[29] McCarthy J., DiSario R., Saraoglu H., (2003), A recursive algorithm for fractionally differencing long data series, Journal of Modern Applied Statistical Methods $2,272-278$.

M.Rubaszek, et al.

CEJEME 2: 151-167 (2010) 
Forecasting the Polish Zloty with Non-Linear Models

[30] Meese R.A., Rogoff K., (1983), Empirical exchange rate models of the 70s. Do they fit out of sample?, Journal of International Economics 14, 3-24.

[31] Meese R.A., Rose A.K., (1990), Nonlinear, nonparametric, nonessential exchange rate estimation, American Economic Review 80, 192-196.

[32] Neely J., Sarno L., (2002), How well do monetary fundamentals forecast exchange rates?, Federal Reserve Bank of St. Louis Review 84, 51-74.

[33] Newey W.K., West K.D., (1987), A simple, positive semi-definite, heteroskedasticity and autocorrelation consistent covariance matrix, Econometrica 55, 703708.

[34] Newey W.K., West K.D., (1994), Automatic lag selection in covariance matrix estimation, Review of Economic Studies 61, 631-653.

[35] Perlin M., (2007), MS_Regress - A package for Markov regime switching models in Matlab, http://www.mathworks.com/matlabcentral/fileexchange/authors/21596

[36] Romer Ch.D., Romer D.H., (2000), Federal Reserve information and the behavior of interest rates, American Economic Review 90, 429-457.

[37] Rumelhart D.E., Hinton D.E., Williams R.J., (1986), Learning internal representation by error propagation, [in:] Parallel Distributed Processing: Exploration in the Microstructure of Cognition [ed.:] Rumelhart D.E., McClelland J.L. , MIT Press, Cambridge MA, 318-362.

[38] Taylor M.P., Peel D.A., Sarno L. (2001) Nonlinear Mean-Reversion in Real Exchange Rates: Toward a Solution to the Purchasing Power Parity Puzzles, International Economic Review 42, 1015-42.

[39] Terasvirta T., Anderson H.M., (1992), Characterizing nonlinearities in business cycles using smooth transition autoregressive models, Journal of Applied Econometrics 7, 119-136.

[40] Wolff Ch., (1987), Time-varying parameters and the out-of-sample forecasting performance of structural exchange rate models, Journal of Business and Economic Statistics 5, 87-97.

[41] Yu L., Wang S., Lai K.K., (2007), Foreign-Exchange-Rate Forecasting with artificial neural networks, Springer, New York. 